\title{
Intermittent Fasting: Current Evidence in Clinical Practice
}

\author{
So Hyun Kang ${ }^{1}$, Young Suk Park ${ }^{1,2}$, Sang-Hoon Ahn ${ }^{1,2, *}$, Hyung-Ho Kim ${ }^{1,2}$ \\ 'Department of Surgery, Seoul National University Bundang Hospital, Seongnam; ${ }^{2}$ Department of Surgery, \\ Seoul National University College of Medicine, Seoul, Korea
}

\author{
Received March 17, 2020 \\ Reviewed March 29, 2020 \\ Accepted April 6, 2020 \\ *Corresponding author \\ Sang-Hoon Ahn \\ https://orcid.org/0000-0001-8827-3625 \\ Department of Surgery, Seoul National \\ University Bundang Hospital, Seoul \\ National University College of Medicine \\ 82 Gumi-ro 173beon-gil, Bundang-gu, \\ Seongnam 13620, Korea \\ Tel: +82-31-787-7106 \\ Fax: +82-31-787-4078 \\ E-mail: viscaria@snubh.org
}

Intermittent fasting (IF), or time-restricted feeding, is an emerging dietary intervention that restricts intake of food and energy for a given period. Not only does this method restrict total caloric intake, it also promotes metabolic homeostasis by supporting circadian feeding rhythms. ${ }^{1,2}$ Only in the most recent 200 years have humans been able to access supplies of vast amounts of food and resources, which has caused a shift in disease patterns, particularly for metabolic syndromes and obesity. The idea that reducing caloric intake can result in evolutionary cellular responses for survival has been an active research topic, ${ }^{3}$ but with the recent public awareness and interest in IF, many clinical studies have been published more recently. On December 2019, de Cabo and Mattson ${ }^{4}$ published a review article that discusses the mechanisms and current clinical evidence for IF. This review article discusses various broad-spectrum benefits of IF, with a positive outlook for more clinical evidence in the future.

The most widely accepted theory behind the primary physiologic response after IF is the switch of energy source from glucose to triglycerides, which has also been termed as the "ketogenic diet." This metabolic switch increases mitochondrial stress resistance, antioxidative defenses, and autophagy while reducing the amount of blood insulin., ${ }^{4,6}$ de Cabo and Mattson ${ }^{4}$ illustrated in their review that stimulating autophagy while inhibiting the mammalian target of rapamycin protein synthesis pathway can lead to removal of oxidatively damaged cells. In a review by Wahl et al., ${ }^{7}$ IF was shown to have positive effects on memory acquisition and cognitive behavior in rodent models. One possible hypothesis for this effect is production of proinflammatory factors with glucose-based diets. ${ }^{8}$ The reduction of these proinflammatory factors may be beneficial for reducing systemic inflammation and oxidative stress factors that play a role in development of atherosclerosis. ${ }^{9}$

Several clinical studies, including some randomized control trials, have been performed to analyze the effects of IF and caloric restriction in a wide range of applications. Although preclinical studies have demonstrated reduction of insulin sensitivity after caloric restriction and periodic fasting, ${ }^{4,10}$ clinical studies have showed inconsistent results. The calorie restriction and cardiometabolic risk (CALERIE) study ${ }^{11}$ is a phase 2 , multicenter, randomized control trial where 218 patients were allocated to either the $25 \%$ caloric restricting group $(\mathrm{n}=143)$ or an ad libitum control group $(\mathrm{n}=75)$. In the restricting group, there was significant loss in body weight and reduction in other cardiometabolic factors such as low-density 
lipoprotein (LDL) cholesterol and blood pressure. Fasting glucose was significantly reduced at the first year, but there was no significant reduction at the second year. However, there was a significant decrease in a surrogate marker for insulin resistance, which was estimated by the homeostatic model assessment for insulin resistance (HOMA-IR). Another study by Marinac et al..$^{8}$ evaluated the frequency and circadian timing of feeding with metabolic syndrome and breast cancer risk in women and showed no association of HOMA-IR with evening calorie intake, eating frequency, or nighttime fasting duration. Recently, Wilkinson et al. ${ }^{2}$ published a singlearm study of 19 patients with metabolic syndrome who were mostly on statin and/or antihypertensive therapy. Patients were restricted to a 10-hour period of feeding and were examined for body composition and other health metrics after 12 weeks. There was significant decrease in body weight, body fat, systolic/diastolic blood pressure, total cholesterol, and LDL-cholesterol. Although there was a trend toward decrease of blood glucose and glycosylated hemoglobin (HbAlc) levels, there was no statistically significant benefit. However, in a subgroup analysis of patients with either elevated fasting glucose $\geq 100 \mathrm{mg} / \mathrm{dL}$ and/or HbAlc $\geq 5.7 \%$, there was a significant reduction in $\mathrm{HbAlc}$ level $(-0.22 \% \pm 0.32 \%$, $P=0.04)$.

IF acts as a stress signal stimulator, preconditioning cells before ischemic tissue injury. ${ }^{4}$ Mauro et al. ${ }^{12}$ compared rodent models with a 3-day water-only fasting, 1 week protein-free fasting, and overnutrition (high-fat diet) diet protocol prior to vascular surgery. Short-term, 3-day fasting before vascular surgery significantly attenuated intimal hyperplasia and reduced ischemia-reperfusion outcomes. A randomized control trial examined bariatric patients who were scheduled to undergo gastric bypass surgery and compared a 14-day very low-calorie diet (VLCD) group with the ad libitum control group. ${ }^{13}$ Although there was no difference in operation time, the number of 30-day complications was higher in the control group than the VLCD group. Abdominal surgery increases in difficulty with increasing body fat composition, and high amounts of visceral fat lead to unclean dissection of the body planes, resulting in an operative field prone to inflammation and fluid collection. In addition, high subcutaneous fat complicates wound closure, resulting in more frequent wound complications.

The possible benefits of IF before elective surgery are currently controversial, especially compared with the Enhanced Recovery After Surgery (ERAS) protocol, which is now being implemented with good outcomes in various types of surgery. ${ }^{14}$ One of the popular components of the ERAS program is reducing preoperative fasting time to 6 hours and providing oral carbohydrate solutions for up to 2 hours before surgery-encouraging preoperative oral nutrition. This theoretically reduces preoperative anxiety, and patients transition into an anabolic state to benefit from postoperative nutrition. ${ }^{15}$ The ERAS protocol is a comprehensive idea for reducing surgical stress to promote faster recovery; however, it does not explore the idea of fasting alone. Thus, the current success of the ERAS protocols may not necessarily be attributable to reduced preoperative fasting, and additional well-designed control studies are needed to elucidate how preoperative fasting affects patient outcome.

Despite all the emerging evidence, there are still some pitfalls and practical difficulties to IF and caloric restriction that are continuously being studied. First, physiologic studies have not yet achieved consensus on the optimal timing for IF. Some studies have used alternative day fasting, and some have used a daily timerestricted schedule. Although studies have shown that IF reduces patient stress ${ }^{4}$ in the long-term, most patients find it stressful and are reluctant to start restricting intake, reducing patient compliance. Worldwide, especially in Korea, many people believe that skipping one of the three meals in a day will result in deterioration of health and nutritional balance. To address such underlying concerns, de Cabo and Mattson ${ }^{4}$ suggested that physicians provide adequate information and continual support to applicable patients.

In this era where food is abundant, scientists must re-evaluate the notion that "more is better" when it comes to nutrition. Despite the amount exploration regarding IF in clinical practice, there are many areas that have not yet been explored in well-designed clinical trials. Therefore, additional research and consideration are needed to optimize patient outcomes.

\section{CONFLICTS OF INTEREST}

The authors declare no conflict of interest. 


\section{AUTHOR CONTRIBUTIONS}

Study concept and design: SHK, SHA; drafting of the manuscript: SHK, SHA; and critical revision of the manuscript: YSP, SHA, HHK.

\section{REFERENCES}

1. Panda S. Circadian physiology of metabolism. Science 2016; 354:1008-15.

2. Wilkinson MJ, Manoogian EN, Zadourian A, Lo H, Fakhouri S, Shoghi A, et al. Ten-hour time-restricted eating reduces weight, blood pressure, and atherogenic lipids in patients with metabolic syndrome. Cell Metab 2020;31:92-104.

3. Ho KY, Veldhuis JD, Johnson ML, Furlanetto R, Evans WS, Alberti KG, et al. Fasting enhances growth hormone secretion and amplifies the complex rhythms of growth hormone secretion in man. J Clin Invest 1988;81:968-75.

4. de Cabo R, Mattson MP. Effects of intermittent fasting on health, aging, and disease. N Engl J Med 2019;381:2541-51.

5. Mattson MP, Longo VD, Harvie M. Impact of intermittent fasting on health and disease processes. Ageing Res Rev 2017; 39:46-58.

6. Di Francesco A, Di Germanio C, Bernier M, de Cabo R. A time to fast. Science 2018;362:770-5.

7. Wahl D, Coogan SC, Solon-Biet SM, de Cabo R, Haran JB, Raubenheimer D, et al. Cognitive and behavioral evaluation of nutritional interventions in rodent models of brain aging and dementia. Clin Interv Aging 2017;12:1419-28.

8. Marinac CR, Sears DD, Natarajan L, Gallo LC, Breen CI, Patterson RE. Frequency and circadian timing of eating may influence biomarkers of inflammation and insulin resistance associated with breast cancer risk. PLoS One 2015;10:e0136240.
9. Moro T, Tinsley G, Bianco A, Marcolin G, Pacelli QF, Battaglia $G$, et al. Effects of eight weeks of time-restricted feeding (16/8) on basal metabolism, maximal strength, body composition, inflammation, and cardiovascular risk factors in resistance-trained males. J Transl Med 2016;14:290.

10. Mattison JA, Colman RJ, Beasley TM, Allison DB, Kemnitz JW, Roth GS, et al. Caloric restriction improves health and survival of rhesus monkeys. Nat Commun 2017;8:14063.

11. Kraus WE, Bhapkar M, Huffman KM, Pieper CF, Krupa Das S, Redman LM, et al. 2 Years of calorie restriction and cardiometabolic risk (CALERIE): exploratory outcomes of a multicentre, phase 2, randomised controlled trial. Lancet Diabetes Endocrinol 2019;7:673-83.

12. Mauro CR, Tao M, Yu P, Treviño-Villerreal JH, Longchamp A, Kristal BS, et al. Preoperative dietary restriction reduces intimal hyperplasia and protects from ischemia-reperfusion injury. $\mathrm{J}$ Vasc Surg 2016;63:500-9.

13. Van Nieuwenhove Y, Dambrauskas Z, Campillo-Soto A, van Dielen F, Wiezer R, Janssen I, et al. Preoperative very low-calorie diet and operative outcome after laparoscopic gastric bypass: a randomized multicenter study. Arch Surg 2011;146: 1300-5.

14. Kang SH, Lee Y, Min SH, Park YS, Ahn SH, Park DJ, et al. Multimodal Enhanced Recovery after Surgery (ERAS) program is the optimal perioperative care in patients undergoing totally laparoscopic distal gastrectomy for gastric cancer: a prospective, randomized, clinical trial. Ann Surg Oncol 2018; 25:3231-8.

15. Fearon KC, Ljungqvist O, Von Meyenfeldt M, Revhaug A, Dejong $\mathrm{CH}$, Lassen $\mathrm{K}$, et al. Enhanced recovery after surgery: a consensus review of clinical care for patients undergoing colonic resection. Clin Nutr 2005;24:466-77. 